\title{
Early efficacy and safety of statin therapy in Korean patients with hypercholesterolemia: Daegu and Gyeongbuk Statin Registry
}

Han Joon Bae ${ }^{1}$, Yun-Kyeong Cho ${ }^{1}$, Hyoung-Seob Park ${ }^{1}$, Hyuck-Jun Yoon ${ }^{1}$, Hyungseop Kim ${ }^{1}$,

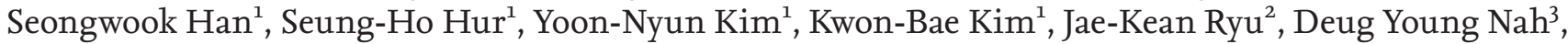
and Chang-Wook Nam ${ }^{1}$

${ }^{1}$ Division of Cardiology, Department of Internal Medicine, Keimyung University Dongsan Hospital, Daegu; 2Division of Cardiology, Department of Internal Medicine, Daegu Catholic University School of Medicine, Daegu; ${ }^{3}$ Division of Cardiology, Department of Internal Medicine, Dongguk University Gyeongju Hospital, Gyeongju, Korea

Received: July 19, 2018

Revised : August 21, 2018

Accepted: August 24, 2018

Correspondence to Chang-Wook Nam, M.D.

Division of Cardiology,

Department of Internal

Medicine, Keimyung University

Dongsan Hospital, 1035

Dalgubeol-daero, Dalseo-gu,

Daegu 42061, Korea

Tel: +82-53-258-7051

Fax: $+82-53-258-7008$

E-mail: ncwcv@dsmc.or.kr
Background/Aims: To date, prospective data are limited on efficacy and safety profiles of statin therapy in Korean hypercholesterolemic patients. Hence, the aim of this study was to evaluate the practice patterns of statin therapy and its efficacy and safety through the prospective Daegu and Gyeongbuk statin registry.

Methods: Statin naïve patients who were prescribed statins according to the criteria of Korean Guidelines for Management of Dyslipidemia were enrolled. Clinical and laboratory evaluations were performed at baseline and at week 8 , where the efficacy was assessed with the same guidelines.

Results: Of 908 patients, atorvastatin and rosuvastatin were most frequently prescribed statins (63.1\% and 29.3\%, respectively). High intensity statins (atorvastatin $40 \mathrm{mg}$ or rosuvastatin $20 \mathrm{mg}$ ) were prescribed in $24.7 \%$ of all patients and in $79.5 \%$ of high and very high risk groups. The total and low density lipoprotein (LDL) cholesterol levels decreased from $203.7 \pm 43.0$ to $140.6 \pm 28.6 \mathrm{mg} / \mathrm{dL}$ and $134.4 \pm 35.7$ to $79.5 \pm 21.3 \mathrm{mg} / \mathrm{dL}$, respectively. The achievement rate of the LDL target goal was 98.6\% in low risk, $95.0 \%$ in moderate risk, $88.1 \%$ in high risk, and $42.1 \%$ in very high risk patients $(59.7 \%$ in overall). There was no significant difference in the efficacy between atorvastatin and rosuvastatin. Adverse events were observed in $12.0 \%$ of patients and led to $1.4 \%$ of treatment cessation.

Conclusions: The efficacy of the usual starting dose of statins in daily practice was relatively insufficient for Korean hypercholesterolemic patients with high or very high risks. Short-term adverse events of statin therapy were not common in Korean patients with a low discontinuation rate.

Keywords: Dyslipidemias; Cholesterol; Hydroxymethylglutaryl-CoA reductase inhibitors

\section{INTRODUCTION}

Recently, the prevalence of cardiovascular disease has increased with the aging population and lifestyle changes in Korea $[1,2]$. The prevalence of hypercholesterolemia, which is a well-known risk factor for cardiovascular disease in patients over 30 years old, has increased from $8.0 \%$ in 2005 to $15.7 \%$ in 2014 , as reported by the Ministry of Health and Welfare in Korea [2]. Statins are effective for the primary and secondary prevention of cardiovascular 
disease by reducing the cholesterol biosynthesis mainly in the liver, through the inhibition of 3-hydroxy-3-methylglutaryl coenzyme A reductase [3-9]. Although statins are usually well tolerated in most patients, several adverse effects including liver and muscle toxicities have been reported in the previous studies [1,3,7,9-15]. There are few prospective data on the efficacy and safety of statin therapy in Korean patients. The aim of this study was to evaluate the practice patterns of statin therapy and its efficacy and safety in patients with dyslipidemia through the prospective Daegu and Gyeongbuk statin registry (DG Statin Registry).

\section{METHODS}

\section{Patient enrollment}

The DG Statin Registry was designed as a prospective observational study enrolling 1,000 patients who were statin-naïve and indicated for statin therapy in Daegu Metropolitan City and Gyeongbuk province of Korea from December 2011 to January 2015. The study was approved by each Institutional Review Committee (DSMC 2011-11-286) and conducted in accordance with the guidelines of the ethics committee at each participating institution. Written informed consent was obtained from all patients.

Inclusion criteria were statin naïve patients who were 30 years or older, in need to use statin therapy, and agreed to a follow-up visit and evaluation after 8 weeks $( \pm 2$ weeks) of statin therapy with informed consent. The exclusion criteria were as follows: (1) an unexplained rise in the creatine phosphokinase (CPK) of $>3$ times the upper limit; (2) alanine aminotransferase (ALT)/aspartate transaminase (AST) values $>1.5$ times the upper limit at baseline; (3) a history of severe or unstable medical or psychological disease, affecting the patient's participation or general condition; and (4) a history of drug or alcohol abuse.

All statins (atorvastatin [ATV], rosuvastatin [RSV], simvastatin, pitavastatin, and fluvastatin) available in the market were registered, and the type of statin prescribed was entirely at the discretion of the individual physician. At the time of the final analysis, the patients' grouping was classified according to the Korean Guidelines for Management of Dyslipidemia [1], and the statin intensi- ty was classified according to the 2013 American College of Cardiology/American Heart Association cholesterol guidelines [12].

\section{Measurements and statistical analyses}

A basic history taking, baseline characteristics, and blood tests were obtained before beginning the statin therapy. A follow-up history taking, and blood tests were repeated at week 8 of the therapy. Patients were requested to fast and avoid any alcohol consumption or cigarette smoking for $>12$ hours before the blood test. The efficacy endpoint was defined as the achievement of the target low density lipoprotein cholesterol (LDL-C) level according to the risk categories of the Korean Guidelines for Management of Dyslipidemia and National Cholesterol Education Program Adult Treatment Panel III (NCEPATP III) guidelines. The secondary efficacy endpoint was the percent change in the lipid parameters including the LDL-C, high density lipoprotein cholesterol (HDL-C), triglyceride, and total cholesterol (TC) levels from baseline to the following outpatient admission.

The safety endpoint was assessed with the incidence of adverse events reported primarily from treatment-related events during the study period. Certain events were classified as safety events of interest, requiring completion of a special electronic case report form. These included an abnormal liver function test classified as an ALT or AST of $>1.5$ times the upper limit, hepatitis with an ALT or AST of $>3$ times the upper limit, myalgia including weakness, general weakness, myopathy, and other muscle symptoms, myopathy as a creatine kinase of $>2$ times the upper limit, and any other complaint of symptoms by the patient after therapy.

Statistical analyses were performed with the SPSS software version 23.0.0.o (IBM Corp., Armonk, NY, USA). Data were reported as frequencies and percentages for dichotomous and categorical variables, and as the mean \pm standard deviation for continuous variables. Dichotomous and categorical variables were assessed using chi-square tests and Fisher's exact tests, and continuous variables were assessed using Student's $t$ tests or the Wilcoxon rank-sum tests, as appropriate, with a significance level of the $p$ value of $<0.05$. 
Table 1. Baseline characteristic

\begin{tabular}{lc}
\hline Characteristic & Value \\
\hline All patients & 908 \\
Demographic & $63.9 \pm 11.6$ \\
Age, yr & $560(61.7)$ \\
Male sex & $24.5 \pm 3.3$ \\
BMI, kg/m ${ }^{2}$ & $475(52.3)$ \\
Smoking & $7(0.8)$ \\
Family history of premature CHD & \\
Comorbidity & $501(55.2)$ \\
\hline Hypertension & $216(23.8)$ \\
\hline Diabetes mellitus & $605(68.1)$ \\
Coronary artery disease & $46(5.1)$ \\
\hline Stroke & $5(0.6)$ \\
\hline Peripheral arterial disease & $5(0.6)$ \\
\hline Carotid disease
\end{tabular}

Values are presented as mean \pm SD or number (\%).

BMI, body mass index; CHD, coronary heart disease.

\section{RESULTS}

From December 2012 to January 2015, 1,000 individuals were initially screened. Ninety-two patients were excluded as follows: 31 due to loss to follow-up, three due to failure to meet the inclusion criteria (elevated baseline liver enzyme), and 58 due to inadequate data. Hence, total of 908 eligible patients were included in the final analysis.

The mean age of the study group was $63.9 \pm 11.6$ years (61.7\% men), and the mean body mass index was $24.5 \pm$ $3.3 \mathrm{~kg} / \mathrm{m}^{2}$. There were $23.8 \%$ of the patients with diabetes mellitus and $68.1 \%$ with obstructive ( $\geq 50 \%$ stenosis) or non-obstructive (<50\% stenosis) coronary artery disease (Table 1). The types of prescribed statins were as follows (Fig. 1): ATV in 63.1\%, RSV in 29.3, simvastatin in 4.23\%, pitavastatin in $2.9 \%$, and fluvastatin in $0.4 \%$.

According to the criteria of the Korean Guidelines for Management of Dyslipidemia [1], the risk levels were stratified into $68.1 \%$ as a very high risk group, $6.6 \%$ as a high risk group, $16 \%$ as a moderate risk group, and $9.4 \%$ as low-risk group. High-intensity statins (ATV $40 \mathrm{mg}$ or RSV $20 \mathrm{mg}$ ) were prescribed in $24.7 \%$ of all patients and were prescribed for $79.5 \%$ of high and very high risk patients. Nearly $73 \%$ of high and very high risk patients

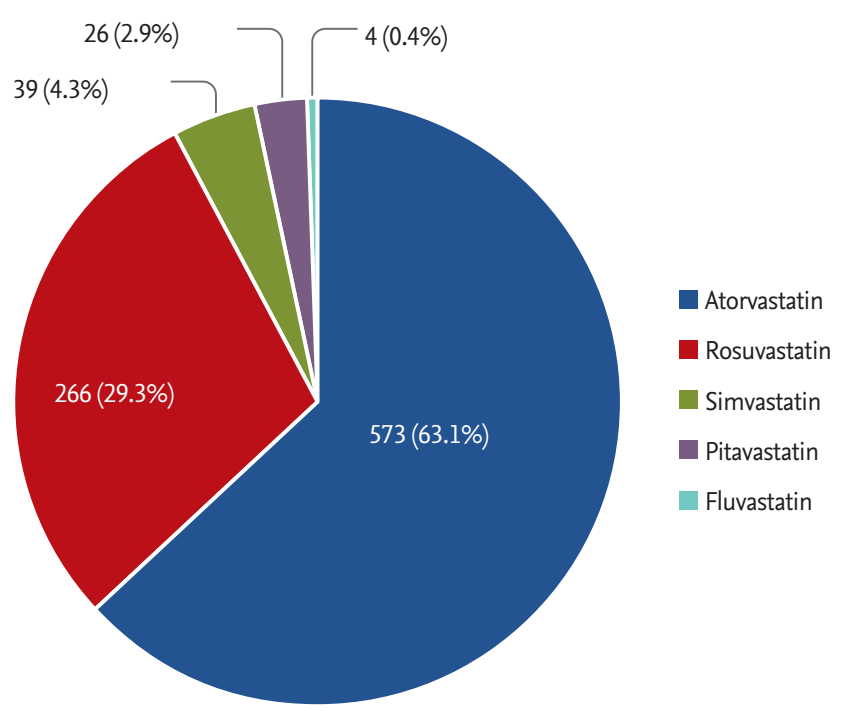

Figure 1. Type of statin. Data are presented as the number (percentage).

were initially prescribed at least one moderate or high intensity statin (Fig. 2A).

If the patients were classified only by the LDL-C levels regardless of their risks, $5.9 \%$ were classified as the very high LDL-C group ( $\geq 190 \mathrm{mg} / \mathrm{dL}$ ), $14.3 \%$ as the high LDL-C group (160 to $189 \mathrm{mg} / \mathrm{dL}$ ), $29.0 \%$ as the borderline high LDL-C group (130 to $159 \mathrm{mg} / \mathrm{dL}$ ), $32.8 \%$ as the near optimal LDL-C group (100 to $129 \mathrm{mg} / \mathrm{dL}$ ), and $18 \%$ as the optimal LDL-C group (<100 mg/dL). Of the patients with a high or very high LDL-C level, moderate intensity statins were used for about $55 \%$ and high intensity statins for more than $30 \%$ (Fig. 2B).

\section{Efficacy}

The mean baseline TC level was $203.7 \pm 43.0 \mathrm{mg} / \mathrm{dL}$, and mean follow-up TC level $140.6 \pm 28.6 \mathrm{mg} / \mathrm{dL}(p<0.001)$. The HDL-C levels were significantly elevated after the statin therapy $(47.7 \pm 11.3$ to $49.0 \pm 11.6 \mathrm{mg} / \mathrm{dL}, p<0.001)$ and the LDL-C levels were significantly reduced after the statin therapy $(134.4 \pm 35.7$ to $79.5 \pm 21.3 \mathrm{mg} / \mathrm{dL}, p<$ 0.001) (Table 2). The distribution of the TC and LDL-C at baseline and at follow-up is shown in Fig. 3 .

The overall achievement of the target LDL-C level was $59.7 \%$ in the DG registry. The achievement rates were 98.6\% in the low risk, $95.0 \%$ in the moderate risk, $88.1 \%$ in the high risk, and $42.1 \%$ in the very high risk patients (Fig. 4). To sum it up, the patients in the high and very 

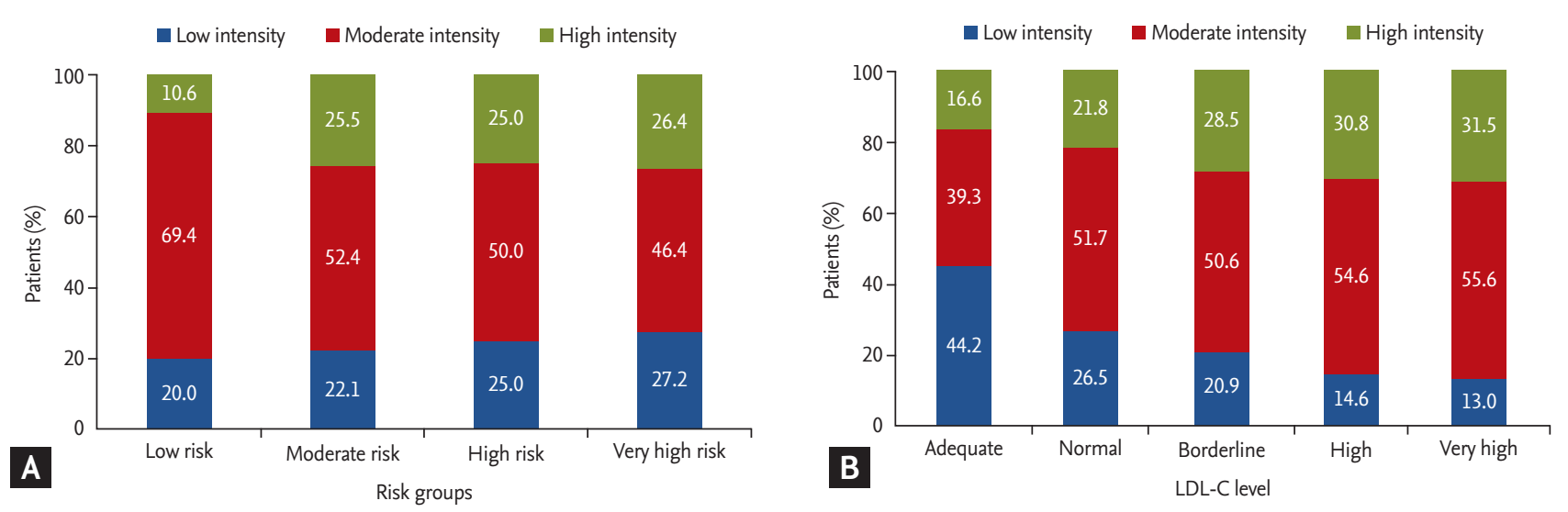

Figure 2. The distribution of the statin therapy according to the (A) risk group. (B) Low density lipoprotein cholesterol (LDL-C). (B) The LDL-C was classified into five groups: adequate < $100 \mathrm{mg} / \mathrm{dL}$, normal 100 to $129 \mathrm{mg} / \mathrm{dL}$, borderline 130 to $159 \mathrm{mg} / \mathrm{dL}$, high 160 to $189 \mathrm{mg} / \mathrm{dL}$, and very high $>190 \mathrm{mg} / \mathrm{dL}$.

Table 2. Laboratory data

\begin{tabular}{|c|c|c|c|}
\hline Characteristic & Baseline & Follow-up & $p$ value \\
\hline \multicolumn{4}{|l|}{ Lipid profile } \\
\hline Total cholesterol, mg/dL & $203.7 \pm 43.0$ & $140.6 \pm 28.6$ & $<0.001$ \\
\hline HDL-C, mg/dL & $47 \cdot 7 \pm 11.3$ & $49.0 \pm 11.6$ & $<0.001$ \\
\hline LDL-C, mg/dL & $134 \cdot 4 \pm 35 \cdot 7$ & $79 \cdot 5 \pm 21.3$ & $<0.001$ \\
\hline $\mathrm{TG}, \mathrm{mg} / \mathrm{dL}$ & $146.6 \pm 103.6$ & $122.2 \pm 63.4$ & $<0.001$ \\
\hline Non-HDL-C, mg/dL & $153 \cdot 7 \pm 41.0$ & $75 \cdot 1 \pm 44 \cdot 5$ & $<0.001$ \\
\hline $\mathrm{AST}, \mathrm{U} / \mathrm{L}$ & $25 \cdot 5 \pm 9.4$ & $25.8 \pm 13.6$ & 0.592 \\
\hline ALT, U/L & $23.9 \pm 11.7$ & $27 \cdot 7 \pm 21.6$ & $<0.001$ \\
\hline BUN, mg/dL & $17 \cdot 3 \pm 7 \cdot 4$ & $16.5 \pm 7.8$ & $<0.001$ \\
\hline Creatinine, mg/dL & $0.91 \pm 0.67$ & $0.94 \pm 0.98$ & 0.159 \\
\hline $\mathrm{CPK}, \mathrm{U} / \mathrm{L}$ & $116.7 \pm 65.0$ & $116.9 \pm 157.5$ & 0.643 \\
\hline $\mathrm{LDH}, \mathrm{U} / \mathrm{L}$ & $371.3 \pm 93.0$ & $383.8 \pm 135 \cdot 3$ & 0.288 \\
\hline hs-CRP, mg/dL & $0.55 \pm 1.70$ & $0.24 \pm 0.94$ & $<0.001$ \\
\hline FBS, mg/dL & $122.0 \pm 41.4$ & $115 \cdot 3 \pm 35.8$ & $<0.001$ \\
\hline Non-DM, mg/dL (n = 692) & $107.1 \pm 22.0$ & $104.2 \pm 18.8$ & 0.037 \\
\hline $\mathrm{DM}, \mathrm{mg} / \mathrm{dL}(\mathrm{n}=216)$ & $155.7 \pm 53.6$ & $140.4 \pm 49.9$ & 0.019 \\
\hline
\end{tabular}

Values are presented as mean $\pm \mathrm{SD}$.

HDL-C, high density lipoprotein cholesterol; LDL-C, low density lipoprotein cholesterol; TG, triglyceride; AST, aspartate transaminase; ALT, alanine aminotransferase; BUN, blood urea nitrogen; CPK, creatine phosphokinase; LDH, lactate dehydrogenase; hs-CRP, high-sensitivity C-reactive protein; FBS, fasting blood sugar; DM, diabetes mellitus.

high risk groups had a lower achievement of the target LDL-C level. Additionally, an achievement rate of more than $30 \%$ of the LDL-C reduction was $81.9 \%, 76.7 \%$, $80.9 \%$, and $72.3 \%$ for the low risk, moderate risk, high risk, and very high risk groups, respectively. Overall, $74.6 \%$ of the patients achieved a $30 \%$ or greater reduc- tion in the LDL-C level.

In addition, despite the short-term follow-up, there was a significant reduction in the high-sensitivity C-reactive protein level after the statin therapy from $0.55 \pm$ 1.70 to $0.24 \pm 0.94 \mathrm{mg} / \mathrm{dL}(p<0.001)$. 

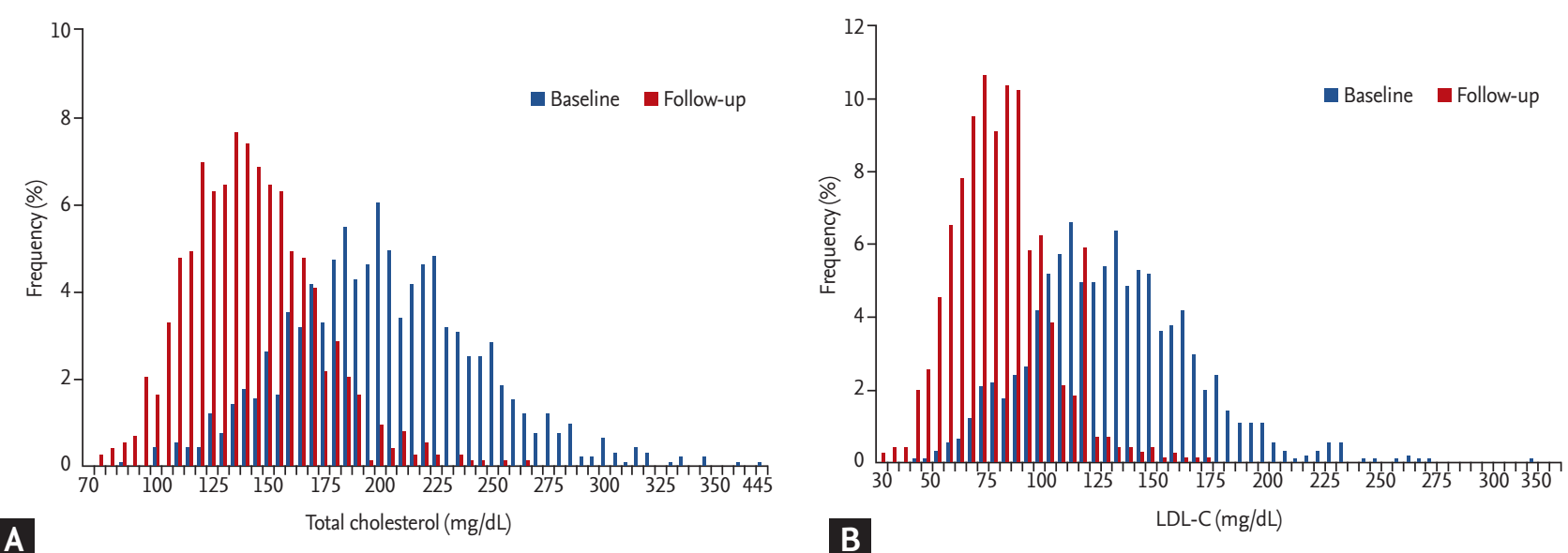

Figure 3. Change in the (A) total cholesterol and (B) low density lipoprotein cholesterol (LDL-C). Data are presented as the number.

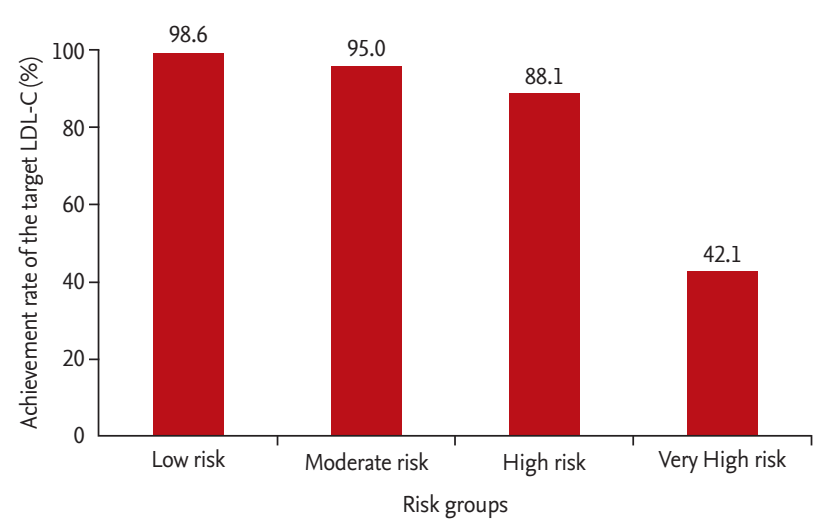

Figure 4. Changes in the low density lipoprotein cholesterol (LDL-C) levels in each risk group.

\section{Safety}

No clinically significant elevations were observed in the follow-up laboratory tests (AST, ALT, lactate dehydrogenase, CPK, and glucose level) at week 8 (Table 2). The incidence of treatment-related adverse events occurring during the study period was $12.0 \%$ (109 patients). Transient elevation of the liver enzymes was reported in 40 patients $(4.4 \%)$ and hepatitis in six patients (0.7\%). Myalgia was reported in 38 patients $(4.1 \%)$ and myopathy in 11 patients (1.2\%). The other adverse events are described in Table 3. There were 13 reports $(1.4 \%)$ of serious adverse events that required cessation of the statin treatment including serious myopathy, intolerable myalgia, and an excessive increase in the liver enzymes ( $\geq 5$ times over the upper normal limit).

\section{Comparison between atorvastatin and rosuvastatin}

As depicted in Fig. 5, the doses of ATV and RSV, which are the two most prescribed statins, increased proportionally to the baseline LDL-C levels. The mean LDL-C values at week 8 were similar across the dosage groups, despite the differences in the baseline level. ATV $10 \mathrm{mg}$ and RSV $5 \mathrm{mg}$ reduced the LDL-C by $31.3 \%$ and $33.9 \%$ ( $p$ $=0.397)$, ATV $20 \mathrm{mg}$ and RSV $10 \mathrm{mg}$ by $39.0 \%$ and $41.2 \%$ $(p=0.331)$, and ATV $40 \mathrm{mg}$ and RSV $20 \mathrm{mg}$ by $42.1 \%$, $42.4 \%(p=0.899)$, respectively. The rate of achieving the target LDL-C level as a primary efficacy endpoint was $100 \%$ in both the ATV and RSV groups in the low risk patients, $96.7 \%$ and $89.7 \%$ in the moderate risk patients, $90.5 \%$ and $87.5 \%$ in the high risk patients, and $38.3 \%$ and $52.1 \%$ in the very high risk patients, respectively. Adverse events of any type occurred in $11.6 \%$ of ATV and $13.2 \%$ of RSV patients $(p=0.787)$.

\section{DISCUSSION}

This study evaluated the usual treatment pattern, efficacy, and safety of statin therapy in Korean patients with dyslipidemia. The main findings were as follows. (1) In daily practice, the commonly prescribed statins were ATV and RSV. (2) The selection of the initial statin dose was dependent on the baseline LDL-C level in daily clinical practice. The statin therapy effectively modified the lipid parameters and those modifications were directly proportional to the statin dose. (3) However, the efficacy 
Table 3. Adverse effects

\begin{tabular}{lc}
\hline Characteristic & Value \\
\hline Number & $109 / 908(12.0)$ \\
Transient elevation of liver enzymes & $40(4.4)$ \\
\hline Hepatitis & $6(0.7)$ \\
Myalgia & $38(4.1)$ \\
\hline Myopathy & $11(1.2)$ \\
\hline Others & $31(3.4)$ \\
\hline Gastrointestinal symptoms problem & $15(1.6)$ \\
\hline Dyspnea & $2(0.2)$ \\
\hline Ecchymosis & $2(0.2)$ \\
\hline Acute tubulo-interstitial nephritis & $1(0.1)$ \\
Cold sweating & $1(0.1)$ \\
\hline Cough & $1(0.1)$ \\
\hline Dizziness & $1(0.1)$ \\
\hline Edema & $1(0.1)$ \\
\hline Headache & $1(0.1)$ \\
\hline Involuntary movement & $1(0.1)$ \\
\hline Urticaria & $1(0.1)$ \\
\hline Bronchiolitis & $1(0.1)$ \\
\hline Chest discomfort & $1(0.1)$ \\
\hline Syncope & $1(0.1)$ \\
\hline
\end{tabular}

Values are presented as number (\%). Abnormal liver function tests were classified as an alanine aminotransferase (ALT) or aspartate transaminase (AST) $>1.5$ times the upper limit; hepatitis as an ALT or AST > 3 times the upper limit; myalgia included weakness, general weakness, myopathy, and other muscle symptoms; myopathy as a creatine kinase $>2$ times the upper limit; and any other symptom complaints by the patient after therapy.

of the usual initial dose of statins was relatively insufficient in the higher risk Korean patients. (4) Early adverse effects of the statin therapy occurred in $12.0 \%$, and in $1.4 \%$ the drug was discontinued. (5) There was no difference between the LDL-C lowering effects and safety issues between ATV and RSV in proportion to the dose.

The biological mechanisms and clinical benefits of statins are well known $[3,4,6]$. Not only do statins modify lipid profiles, but also stabilize atherosclerotic plaque by ameliorating endothelial dysfunction, reducing the inflammatory response, and diminishing the thrombogenicity $[3,4,10]$. Large clinical trials also demonstrated that statin therapy is an effective measure for primary and secondary prevention of cardiovascular events [15-

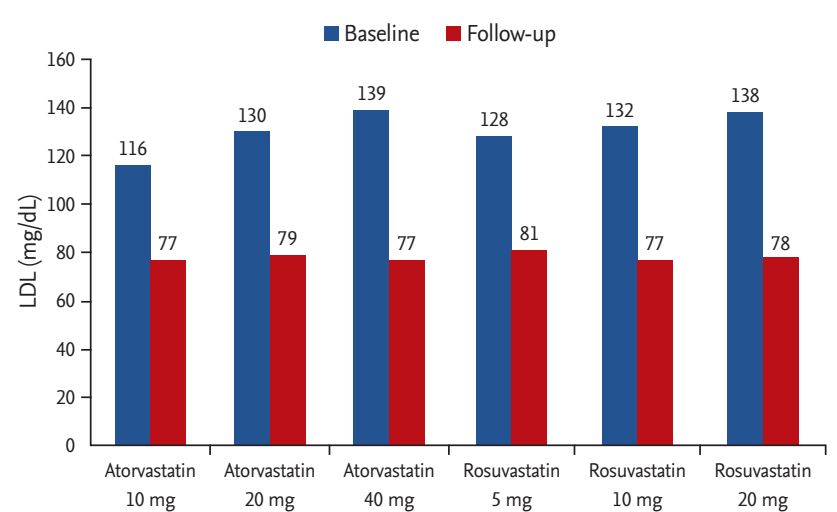

Figure 5. Baseline and follow-up low density lipoprotein (LDL) cholesterol levels according to the dose of the statin.

17]. Although current Western guidelines no longer set a target LDL-C level for statin therapy [18], the 2015 Korean guidelines for the treatment of dyslipidemia recommend to first classify patients by cardiovascular risk factors, and then determine the target goal of the LDL-C level [1]. Similar to the Korean guidelines, the recent European lipid guidelines have also recommend LDL-C targets of $<70$ or $<100 \mathrm{mg} / \mathrm{dL}$ in patients with a very high or high cardiovascular risks, respectively [19,20]. Recently updated Western guidelines are based on studies conducted in non-Asian populations. Despite the short-term follow-up of 8 weeks, given that a previous study has shown Asians are more susceptible to statin therapies than Caucasians [21], this study is valuable in that it investigated changes in lipid parameters and adverse events that arose from the initial statin therapy in a large cohort comprised of Korean patients

In the current registry, only $5.9 \%$ of patients started statins only based on high baseline cholesterol levels (LDL-C > $190 \mathrm{mg} / \mathrm{dL}$ ). Most patients were classified to high or very high risk groups $(74.7 \%, \mathrm{n}=678$ ) according to their underlying cardiovascular risk factors such as proven atherosclerotic cardiovascular diseases or diabetes. Similar to the recent Western guidelines that recommend ATV and RSV as being highly effective for a first-line treatment for hypercholesterolemia [13], the ATV and RSV were mainly prescribed $63.1 \%$ and $29.3 \%$ of the time, respectively. Therefore, the first selected statins for the treatment of hypercholesterolemia in Koreans did not generally differ from the current Western guidelines' recommendations. As shown in Fig. 5, 
various starting doses are given to patients in the routine clinical practice in Korea according to the baseline LDL-C levels and a reduction in the absolute value of the LDL-C related to a higher dose of statins, similar to the previous studies $[1,22]$.

Another important finding was the efficacy of the initial dose of the statin. The overall achievement of the LDL-C target goal by the current Korean guidelines [1] was relatively low (59.7\%) in the DG registry. Similar results were also found in the CEntralized Pan-European survey on tHE Under-treatment of hypercholeSterolemia (CEPHEUS) Pan-Asian survey in which the LDL-C goals were reached in $34.9 \%$ (LDL-C $<70 \mathrm{mg} / \mathrm{dL}$ ) of patients and $55.4 \%(\mathrm{LDL}-\mathrm{C}<100 \mathrm{mg} / \mathrm{dL})$ of the patients with high or very high cardiovascular risks, respectively [12]. In the current study, only $45.9 \%$ of the patients in the high or very high risk groups achieved an appropriate LDL-C goal, while $96.4 \%$ of the patients in the low and moderate risk groups achieved the goal. Although moderate intensity statins and high intensity statins were prescribed $80.0 \%$ and $77.9 \%$ respectively, in the low and moderate risk groups, only $75.0 \%$ and $72.8 \%$ were prescribed in the high and very high risk groups, respectively. Proven to be highly effective, ATV or RSV was prescribed in most of the very high risk patients (96.3\%). However, high-intensity statin doses were prescribed to only $26.4 \%$ of very high risk patients $(29.0 \%$ of ATV or RSV). Whether the target of the statin therapy was set in the absolute value of the LDL-C or \% reduction, the initial dose of statin was relatively insufficient to reach the target goal in the higher risk groups regardless of Asian's susceptibility issues to statin therapies. Unlike the guidelines for hypercholesterolemia management in the United States and Europe, the 2015 Korean Guidelines for Management of Dyslipidemia exhibit a difference in the target LDL-C level according to the risk groups $[1,12,13,23]$. Although the controversy over which guidelines are desirable is unclear and should be further assessed in the future, at this time it is necessary to select higher starting doses of statins for the initial treatment in patients with higher risks.

In addition to the lower prescription rate of high dose statins, the achievement of the LDL-C goal in patients with high dose statins was only $63.2 \%$. Therefore, a non-statin therapy, including ezetimibe or a proprotein convertase subtilisin/kexin 9 (PCSK 9) inhibitor, may be considered in adjunction to patients starting on high dose statins [24-27].

The incidence of adverse events of statin therapy was relatively low at $12.0 \%$ with a discontinuation rate of $1.4 \%$ in the DG registry. The low incidence might be related to the short-term follow-up of the design of the current study. An abnormal elevation of the liver function test and myalgia were the most common adverse effects. In most cases, they were asymptomatic mild elevations of the liver enzymes, which did not cause serious liver damage, such as an increase in the bilirubin level or synthetic dysfunction. When a statin-treated patient shows elevated liver enzymes, one should not be inclined to think that the elevated liver enzymes are due to the statin therapy. Rather, the onset of increased liver enzymes should prompt the clinician to engage in a careful and systematic evaluation (history and physical examination), with consideration of all potential etiologies [14,15]. Myotoxicity is another major complication of statin therapy that is mostly presented as myalgia. In the DG registry, myalgia was reported with an occurrence of $4.1 \%$, which was similar to the other observational cohorts $[14,15]$. Further, there was no statistical difference in the adverse events between ATV and RSV. Myalgia was slightly higher with RSV, but it was not statistically significant (ATV 3.4\%, RSV 6.8\%, p = 0.080). There was no difference in the occurrence of other adverse effects. As emphasized in the United States recommendations, education on adverse events will be necessary in prior to the use of these medicines [13]. It is also important to take notes of medical history during the outpatient visits after starting the medication.

The Justification for the Use of Statins in Prevention: An Intervention Trial Evaluating Rosuvastatin (JUPITER) and other studies reported an increased risk of developing diabetes mellitus in patients on statin therapy [28]. Lipophilic statins are reported to increase the risk of elevated serum glucose levels, especially with higher doses. However, in the DG registry, the serum fasting glucose level decreased after the first statin therapy. This result may be related to the design of the study with a short-term follow-up period, and the educational effects on personal will be needed to improve one's lifestyle after receiving the first statin therapy. A recent Japanese study reported that the change in the glucose level was not significant during the first 3 months, but was 
significant between 6 to 12 months, resulting in a slight rise in the hemoglobin Aic [29]. Therefore, the current study could not show the long-term effects of statins on the fasting plasma glucose level.

There are several limitations of the current study. First, this study was a region based observational study, which could result in a selection bias. Therefore, this data is not fully representative of Korean patients requiring statin treatment. This point also should be described as another limitation of this study. However, with a wide inclusion criteria, this study could directly reflect the prescription patterns of the real clinical practice and evaluate the efficacy and safety of the current statin treatment. Second, due to the design of the current study with a short-term follow-up, the long-term effects of the actual statin therapy could not be assessed. Third, because a core lab analysis was not performed, some variations between each center could have affected the results. However, all the patients were enrolled from tertiary referral centers. Therefore, the minor variations in the laboratory tests might be neglectable. Lastly, because this was an observational study and not a randomized controlled study, there is a limitation in the direct comparison of the ATV and RSV effects.

In conclusion, the most commonly prescribed statins in daily practice were ATV and RSV in up to $92 \%$ of the patients. The efficacy of the usual starting dose of statins in daily practice was relatively insufficient in high risk Korean patients. Although the adverse events of statins were not common in Korean patients, careful monitoring should be warranted.

\section{KEY MESSAGE}

1. In the real world, a reduction in the low density lipoprotein cholesterol (LDL-C) of $>30 \%$ was achieved in $74.6 \%$. However, the achievement rate of the LDL-C target goal was insufficient (59.7\%).

2. The early side effects of statins were not rare and were about $12 \%$. However, the serious side effects that caused cessation of the statin therapy were only $1.4 \%$, which might be an acceptable range in patients with some cautions.

3. There was no differences in the LDL-C lowering efficacy between atorvastatin and rosuvastatin, which are regarded as highly effective statins commercially.

4. The efficacy of the usual starting dose of statins in daily practice was relatively insufficient in higher risk Korean patients. Therefore, in early statin therapy, a higher dose of statins should be recommended in the patients in the higher risk groups.

\section{Conflict of interest}

No potential conflict of interest relevant to this article was reported.

\section{Acknowledgments}

This study was supported by the Cardiology Society of Daegu-Gyeongbuk, Republic of Korea.

\section{REFERENCES}

1. Committee for the Korean Guidelines for the Management of Dyslipidemia. 2015 Korean guidelines for the management of dyslipidemia: executive summary (English translation). Korean Circ J 2016;46:275-306.

2. Gerber RT, Ielasi A, Al-Lamee R, et al. Long-term follow-up of multivessel percutaneous coronary intervention with drug-eluting stents for de novo lesions with correlation to the SYNTAX score. Cardiovasc Revasc Med 2011;12:220-227.

3. Moon GJ, Kim SJ, Cho YH, Ryoo S, Bang OY. Antioxidant effects of statins in patients with atherosclerotic cerebrovascular disease. J Clin Neurol 2014;10:140-147.

4. Gaw A. The care gap: underuse of statin therapy in the elderly. Int J Clin Pract 2004;58:777-785.

5. LaRosa JC, He J, Vupputuri S. Effect of statins on risk of coronary disease: a meta-analysis of randomized controlled trials. JAMA 1999;282:2340-2346.

6. Stancu C, Sima A. Statins: mechanism of action and effects. J Cell Mol Med 2001;5:378-387.

7. Thom T, Haase N, Rosamond W, et al. Heart disease and stroke statistics: 2006 update: a report from the American Heart Association Statistics Committee and Stroke Statistics Subcommittee. Circulation 2006;113:e85-e151.

8. Ko DT, Mamdani M, Alter DA. Lipid-lowering therapy 
with statins in high-risk elderly patients: the treatment-risk paradox. JAMA 2004;291:1864-1870.

9. Bellosta S, Paoletti R, Corsini A. Safety of statins: focus on clinical pharmacokinetics and drug interactions. Circulation 2004;109(23 Suppl 1):III5O- III57.

10. Jones PH, Davidson MH, Stein EA, et al. Comparison of the efficacy and safety of rosuvastatin versus atorvastatin, simvastatin, and pravastatin across doses (STELLAR* Trial). Am J Cardiol 2003;92:152-160.

11. European Association for Cardiovascular Prevention \& Rehabilitation, Reiner Z, Catapano AL, et al. ESC/EAS guidelines for the management of dyslipidaemias: the task force for the management of dyslipidaemias of the European Society of Cardiology (ESC) and the European Atherosclerosis Society (EAS). Eur Heart J 2011;32:1769-1818.

12. Stone NJ, Robinson JG, Lichtenstein AH, et al. 2013 ACC/ AHA guideline on the treatment of blood cholesterol to reduce atherosclerotic cardiovascular risk in adults: a report of the American College of Cardiology/American Heart Association Task Force on Practice Guidelines. J Am Coll Cardiol 2014;63(25 Pt B):2889-2934.

13. Mancini GB, Baker S, Bergeron J, et al. Diagnosis, prevention, and management of statin adverse effects and intolerance: Canadian Consensus Working Group update (2016). Can J Cardiol 2016;32(7 Suppl):S35-S65.

14. Bays H, Cohen DE, Chalasani N, Harrison SA; The National Lipid Association's Statin Safety Task Force. An assessment by the Statin Liver Safety Task Force: 2014 update. J Clin Lipidol 2014;8(3 Suppl):S47-S57.

15. Downs JR, Clearfield M, Weis S, et al. Primary prevention of acute coronary events with lovastatin in men and women with average cholesterol levels: results of AFCAPS/TexCAPS. Air Force/Texas Coronary Atherosclerosis Prevention Study. JAMA 1998;279:1615-1622.

16. West of Scotland Coronary Prevention Study: identification of high-risk groups and comparison with other cardiovascular intervention trials. Lancet 1996;348:1339-1342.

17. Jacobson TA, Ito MK, Maki KC, et al. National lipid association recommendations for patient-centered management of dyslipidemia: part 1. Full report. J Clin Lipidol 2015;9:129-169.

18. Jellinger PS, Handelsman Y, Rosenblit PD, et al. American Association of Clinical Endocrinologists and American College of Endocrinology Guidelines for management of dyslipidemia and prevention of cardiovascular disease. Endocr Pract 2017;23(Suppl 2):1-87.
19. Wang P. Statin dose in Asians: is pharmacogenetics relevant? Pharmacogenomics 2011;12:1605-1615.

20. Martineau P, Gaw A, de Teresa E, et al. Effect of individualizing starting doses of a statin according to baseline LDL-cholesterol levels on achieving cholesterol targets: the achieve cholesterol targets fast with atorvastatin stratified titration (ACTFAST) study. Atherosclerosis 2007;191:135-146.

21. Park JE, Chiang CE, Munawar M, et al. Lipid-lowering treatment in hypercholesterolaemic patients: the CEPHEUS Pan-Asian survey. Eur J Prev Cardiol 2012;19:781794.

22. American Diabetes Association. Standards of medical care in diabetes: 2016. Diabetes Care 2016;39 (Suppl 1):s6o-s71.

23. National Cholesterol Education Program (NCEP) Expert Panel on Detection, Evaluation, and Treatment of High Blood Cholesterol in Adults (Adult Treatment Panel III). Third report of the National Cholesterol Education Program (NCEP) expert panel on detection, evaluation, and treatment of high blood cholesterol in adults (Adult Treatment Panel III) final report. Circulation 2002;106:3143-3421.

24. Sabatine MS, Giugliano RP, Keech AC, et al. Evolocumab and clinical outcomes in patients with cardiovascular disease. N Engl J Med 2017;376:1713-1722.

25. Cho YK, Hur SH, Han CD, et al. Comparison of ezetimibe/simvastatin 10/20 $\mathrm{mg}$ versus atorvastatin $20 \mathrm{mg}$ in achieving a target low density lipoprotein-cholesterol goal for patients with very high risk. Korean Circ J 2011;41:149-153.

26. Nam CW, Kim DS, Li J, et al. Efficacy and safety of alirocumab in Korean patients with hypercholesterolemia and high cardiovascular risk: subanalysis of the ODYSSEY-KT study. Korean J Intern Med 2018 Sep 1 [Epub]. https://doi. org/10.3904/kjim.2018.133.

27. Cannon CP, Blazing MA, Giugliano RP, et al. Ezetimibe added to statin therapy after acute coronary syndromes. N Engl J Med 2015;372:2387-2397.

28. Ridker PM. The JUPITER trial: results, controversies, and implications for prevention. Circ Cardiovasc Qual Outcomes 2009;2:279-285.

29. Ogawa H, Matsui K, Saito Y, et al. Differences between rosuvastatin and atorvastatin in lipid-lowering action and effect on glucose metabolism in Japanese hypercholesterolemic patients with concurrent diabetes. Lipid-lowering with highly potent statins in hyperlipidemia with type 2 diabetes patients (LISTEN) study. Circ J 2014;78:2512-2515. 